\title{
La Escuela Graduada en España. Similitudes con el actual modelo educativo
}

\author{
The Escuela Graduada Model. Similarities with the current educational \\ model in Spain
}
A Escola Graduada na Espanha. Similaridades com o atual modelo
educativo

\author{
Óscar Navarro Martínez ${ }^{1}$ \\ Universidad de Castilla-La Mancha, España
}

Recepción: 12/03/2018

Evaluación: 05/05/2018

Aceptación: 29/06/2018

Artículo de Investigación - Reflexión

DOI: $10.19053 / 01227238.7394$

\section{RESUMEN}

La situación del sistema educativo español a finales del siglo XIX repercutió en la necesidad de modernizar los modelos vigentes en aquella época. En ese contexto irrumpe en el país un nuevo planteamiento educativo, la Escuela Graduada. Aporta nuevas técnicas, estructuras y métodos novedosos para intentar paliar la posición rezagada de la educación en España en comparación con otros países europeos. Ante todo, surgió la necesidad de abrirse a todos los niveles sociales, pues en aquellas fechas la educación estaba reducida únicamente a los sectores más favorecidos. Se pretende dar una visión general de lo que supuso la introducción de este modelo y lo que significó para el sistema escolar español durante el primer tercio del pasado siglo. La oposición de algunos sectores del ámbito docente y la pugna con la Escuela Unitaria exige contrastar ambos modelos. Se analizan los aspectos más importantes del modelo graduado, donde apareció un nuevo planteamiento educativo que solo se podía llevar a cabo con una escuela moderna y adaptada a las circunstancias de la época. Además, se compara con el modelo educativo actual, comprobando la vigencia de muchas de las mejoras introducidas.

Palabras clave: Sistemas educativos; experiencia educativa; Escuela Graduada; Escuela Unitaria.

1 Doctor en Pedagogía por la UCLM, licenciado en Ciencias de la Educación por la UNED y maestro de Educación Física por la UCM. Ha trabajado como maestro desde 1997. Actualmente trabaja en la Facultad de Educación de Ciudad Real de la Universidad de Castilla-La Mancha. Sus principales áreas de interés son la elaboración y aplicación de materiales multimedia con alumnos de la etapa de Educación Primaria, la utilización de sistemas LMS y prácticas de e-learning, con alumnos sin y con necesidad específica de apoyo educativo. Correo electrónico: oscar.navarro@uclm.es 


\section{ABSTRACT}

The situation of the Spanish educational system at the end of the 19th century led to the need to modernize the models in force at that time. In this context, a new educational approach emerge in the country, the Escuela Graduada. This model provides new techniques, structures and innovative methods to mitigate the lagging position of education in Spain compared to other European countries. First of all, there was a need to open up to all social levels, since at that time education was limited only to the most privileged sectors.

We intend to offer a general view about the implications of the introduction of this model and what it meant for the Spanish school system during the first third of the last century. The opposition of some teaching sectors and the struggle with the Unitarian School allow us to contrast both models. We analyzed the most important aspects of the Escuela Graduada model, from which arose a new educational approach that could only be carried out with a modern school adapted to the circumstances of the time. In addition, we compared it with the current educational model, in order to verify the validity of those improvements.

Keywords: Educational systems; educational experience; Escuela Graduada; Unitary School system.

\section{RESUMO}

A situação do sistema educativo espanhol nos fins do século XIX repercutiu na necessidade de modernizar os modelos vigentes naquela época. Nesse contexto, irrompe no país uma nova abordagem educativa, a Escola Graduada. Contribui com novas técnicas, estruturas e métodos inovadores com a intenção de aliviar a posição atrasada da educação na Espanha em comparação com outros países europeus. Primeiro de tudo, surgiu a necessidade de abrir-se a todos os níveis sociais, pois naquele tempo a educação estava reduzida unicamente aos setores mais favorecidos. Pretende-se apresentar uma visão geral do que esse modelo implicou e o que significou para o sistema escolar espanhol durante o primeiro terço do século passado. A oposição de alguns setores do âmbito docente e a luta com a Escola Unitária exige contrastar ambos os modelos. Analisam-se os aspectos mais importantes do modelo graduado, donde apareceu um novo planejamento educativo que somente se podia levar a cabo com uma escola moderna e adaptada às circunstâncias da época. Ademais, se compara com um modelo educativo atual, comprovando a vigência de muitas das melhoras introduzidas.

Palavras-chave: Sistemas educativos; experiência educativa; Escola Graduada; Escola Unitária.

\section{INTRODUCCIÓN}

Durante el final del siglo xIX y principios del $\mathrm{xx}$ se produjeron importantes cambios en el contexto social, tanto del viejo continente como en toda América. Desde el punto de vista pedagógico, siempre se han establecido puentes entre ambos lados del océano Atlántico, aunque se debe tener en cuenta las diferencias evidentes al desarrollarse en entornos muy diferentes ${ }^{2}$. Este hecho implicó una importante transformación del ámbito educativo, tal como se concebía por

2 Ana Guil y Sara Vera, "Entre Europa y América Latina: Mercedes Rodrigo, psicopedagoga pionera”, Revista Historia de la Educación Latinoamericana, 13, n. ${ }^{\circ} 17$ (2012): https://doi.org/10.19053/01227238.1598. 
entonces. Esta situación obligó a modificar sus estructuras y adaptarlas a la nueva realidad sociocultural.

La finalidad de la presente investigación es realizar un análisis de las semejanzas y diferencias de la Escuela Graduada con el actual marco educativo. También se pretende profundizar en diversos aspectos de este modelo, comparándolo con la Escuela Unitaria, que fue el sistema más extendido hasta esas fechas.

Como metodología se utilizó la investigación historiográfica, tanto diacrónica como sincrónica. Se abordó la evolución de la Escuela Graduada implantada a lo largo de las últimas décadas del siglo xix y el primer tercio del siglo xx. También se realizó una comparación con el modelo imperante hasta el momento, la Escuela Unitaria. Fue posible considerar dos prototipos que, con una filosofía distinta, pueden ser complementarios dependiendo del contexto y la situación concreta donde se lleva a cabo el proceso educativo. Si bien ambos modelos han perdurado, el graduado ha tenido una mayor predominancia.

Se comenzará con un análisis de la aparición de la Escuela Graduada en España, ahondando en los problemas surgidos y comparándola con el actual marco educativo. Posteriormente se profundiza en la organización escolar de este modelo y uno de sus elementos innovadores fundamentales, los espacios escolares. Luego se hace una comparación de los dos prototipos imperantes en el ámbito educativo, las escuelas Graduada e Unitaria. Por último se exponen las conclusiones generales del artículo.

\section{La Escuela Graduada en España}

En el marco de la educación elemental y básica de cualquier país, la graduación escolar representa la principal innovación del siglo xIx. Durante todo este siglo se fue perfilando la organización y estructuración de este modelo, con algunos antecedentes en el sistema lancasteriano. El impulso definitivo hacia el modelo graduado estuvo condicionado por la gran afluencia de alumnado al entorno educativo debido a la concentración de la población a los, cada vez más crecientes, grandes núcleos urbanos ${ }^{3}$.

En España, la situación de la escuela pública en el siglo XIX presentaba un "panorama desolador" en todos los aspectos, en lo referente a lo material, espacios, recursos ${ }^{4}$. La formación del profesorado se antojaba muy deficiente, con escasas retribuciones y una tasa de absentismo muy alta ${ }^{5}$.

Si se examina el concepto e historia de la Escuela Graduada, se vislumbra que este movimiento conlleva mucho más contenido implícito de lo que se puede suponer en un primer momento. No se reduce simplemente a la implantación de graduación en la escuela, pues al profundizar se observa que llevaba incluida

3 Arturo de la Orden, "La escuela en transición", Revista de educación, n. 266 (1981): 133-149.

4 Ministerio de Instrucción Pública y Bellas Artes, Planos modelos de escuelas graduadas con presupuestos reducidos (Madrid: Imprenta del Colegio Nacional de Sordomudos y Ciegos, 1912).

5 Pedro L. Moreno, "Renovación pedagógica y compromiso social en la edad de plata de la Pedagogía española: Félix Martí Alpera (1898-1920)”, Revista española de pedagogía, n. ${ }^{\circ} 231$ (2005): 203-222. 
una reforma bastante profunda del sistema educativo de la época implantado en España. Antes de seguir adelante se debe distinguir entre Escuela Graduada y graduación escolar. La primera engloba a la segunda, pues a lo largo de la historia se han realizado varias formas de graduación escolar en las que la Escuela Graduada solo es una de ellas.

\section{Aparición de la Escuela Graduada}

Se extiende desde el siglo XIX, aunque podríamos encontrar precedentes desde el siglo xIv. En España se establece legalmente en 1898, de modo experimental y en pleno periodo de crisis. Muchas de sus bases se acabarían imponiendo y ahora se ve este período como muy fructífero en la modernización del sistema educativo. Sin embargo, el camino fue muy duro y salió mal en muchas ocasiones debido a las reticencias a los cambios de distintos sectores. Puede parecer a primera vista que la implantación de la Escuela Graduada fue un fracaso, pues no llegó a generalizarse, pero no se puede negar que marcó una época de modernización del planteamiento educativo, aunque estaba predestinado en cierta forma al "fracaso" como otras leyes educativas (Ley Moyano de 1857 o The 1944 Education Act en Inglaterra). No porque estuvieran mal planteadas, sino por la imposibilidad en ese momento de aportar los recursos materiales y humanos necesarios para llevarlas a cabo.

La principal innovación que propone la Escuela Graduada es la estructura en distintos grados, estableciendo grupos homogéneos. Pero destacamos otros dos elementos que tuvieron gran relevancia, la arquitectura de los edificios (buscaba funcionalidad y capacidad de más alumnos) y la coordinación entre los profesores. Este último es un aspecto muy importante en la actualidad y conserva mucha vigencia.

Otras consecuencias e implicaciones que tuvieron relevancia dentro de la Escuela Graduada son las siguientes:

- Existencia de maestros encargados de las enseñanzas especiales (música, dibujo, educación física, talleres). Hasta entonces, un único maestro se encargaba de la formación de los alumnos en todas las áreas que se impartían en el colegio. Esta línea de actuación ha continuado en la formación del profesorado hasta la aparición, en un primer momento, de las especialidades, y actualmente de las menciones (con el Plan Bolonia).

- Nueva concepción educativa y organizativa, nuevas disciplinas tanto o más importantes que las materias tradicionales. Igual de significativas son áreas más instrumentales (lenguaje o cálculo) que otras que permiten el desarrollo del individuo y que ayudan a una formación integral de la persona. En realidad, este concepto es innovador para la época comparado con los sistemas anteriores, pero ya tuvo una gran relevancia en la antigua Grecia.

- Diversificación de espacios, actividades, usos y personas. Se les da más importancia a los espacios de usos comunes o comunitarios. Se busca la 
funcionalidad de los espacios, sin recodos y bien vigilados. Esto permitirá una mayor eficacia de los maestros en el campo profesional, favoreciendo su uso y realización de actividades.

- Aparecen las Juntas de Profesores, que pueden corresponder con los actuales Claustros. En la Escuela Unitaria no existía apenas relación entre maestros, y aunque hubiera alguna escuela con varias unidades, funcionaban de modo independiente. En la actualidad también se trabaja desde un punto de vista colectivo y colaborativo, estableciendo como un principio fundamental el trabajo en equipo.

- Aparecen los papeles de director y secretario, que empiezan a plantear una jerarquía entre profesores que antes estaban al mismo nivel. En el presente no es posible entender un colegio sin estos órganos, que se amplían con otros órganos unipersonales como el jefe de estudios.

- Se establecen los Consejos Escolares, planteando una apertura de la escuela a la sociedad. Han pasado por distintas acepciones tanto en España como en otros países: Juntas Locales, Consejos Locales, Consejos Provinciales. En la actualidad no se concibe una comunidad educativa que no esté integrada por distintos agentes educativos (profesorado, alumnado, familias, ayuntamiento...).

- Se intenta generalizar la especialización de los profesores en un grado, en lugar de atender a un abanico más amplio de edades en el que resulta difícil trabajar conjuntamente con todos los alumnos.

No hay que confundir la Escuela Graduada con edificios que tienen varias aulas, pues no siempre que hay un colegio de estas características implica que sigue este modelo. Hay centros docentes de más de un aula, pero que se podría considerar como un grupo de Escuelas Unitarias. En definitiva se fomenta una mayor especialización en la enseñanza.

\section{Problemas surgidos}

De estas consecuencias se destaca la última. Se comenta el intento del nuevo sistema de anular la desigualdad existente en el alumnado de las escuelas unitarias. Hasta entonces un mismo docente debía enseñar a alumnos de edades muy variadas, donde la diversidad del alumnado establecía muchas limitaciones y marcaba las líneas a seguir. Sin embargo ahora se parte de supuestos y criterios de organización totalmente distintos, que condujeron a un cambio no solo en lo visible, sino en la concepción de la enseñanza.

La inclusión de estas novedades despertó reticencias dentro del sector educativo de la época y esta posición reaccionaria se ha repetido a lo largo de todas las reformas introducidas en educación a lo largo de la historia. En los últimos treinta años se ha podido comprobar en las distintas leyes aprobadas, como la Ley Orgánica 1/1990, del 3 de octubre, de ordenación general del sistema educativo (LOGSE), la Ley Orgánica 2/2006, del 3 de mayo, de educación (LOE), y más recientemente la Ley Orgánica 8/2013, del 9 de diciembre, para la mejora de la 
calidad educativa (LOMCE). Esta última ha creado mucha polémica, hasta el punto de paralizarse parcialmente y entrar en vigor solo algunos de sus apartados.

Aunque hayan pasado más de cien años desde que se propuso la implantación de la Escuela Graduada, se puede ver que tanto entonces como en la actualidad se tienen reservas hacia los nuevos cambios y una nueva organización del ámbito educativo. Aparecieron importantes críticas en cuanto a la elección de directores. Curiosamente también este cambio ha tenido polémica y ya se contemplaba en la LOE, y luego en la LOMCE, donde cambia el sistema de elección dando más autoridad a la administración y restándosela a los agentes educativos. Por supuesto han surgido las desaprobaciones a este nuevo sistema. Hace más de un siglo hubo más problemas, no solo en la elección, sino en las funciones y deberes, pues se trataba de una figura que no había existido y que estaba dirigida a cambiar la organización de los centros educativos.

Fueron cambios considerables que, como se podía prever, levantaron polémicas y agravios entre los docentes, que antes se mantenían en el mismo escalón jerárquico, teniendo por encima únicamente a la figura del inspector. En este punto señalar que el malestar creado por estas situaciones se sumó a las cada vez más frecuentes visitas "incómodas" del inspector, a las cuales no estaban acostumbrados.

En general, la atención a un número mucho mayor de alumnos exigió la necesidad de plantear un nuevo modelo organizativo a nivel administrativo. Se exigía la necesidad de una figura con una labor "puramente mecánica", para que todo el personal docente quedara eximido de trámites burocráticos, que en definitiva perjudicaban su adecuado "trabajo esencial, que es el pedagógico" 6 .

Hasta ahora se han comentado las expectativas que se tenían de la Escuela Graduada y de las reticencias que se presentaron. Este rechazo por parte de los distintos sectores que intervenían en la educación no fue, por supuesto, el único problema. Ante todo, la dificultad más sustancial fue la limitación de los recursos económicos. Por desgracia, son situaciones cíclicas que también tienen su reflejo en el momento actual. En la última década hemos sido testigos de un gran recorte en los recursos educativos, aunque no es comparable a la situación de principios del siglo pasado.

Una reforma que pretende variar los métodos de enseñanza, la construcción de nuevas instalaciones que puedan responder a las necesidades creadas y requiere un número mayor de profesorado (debido al aumento considerable del alumnado a escolarizar) no puede llevarse a cabo si no existe un importante respaldo financiero. Por desgracia para los impulsores de la Escuela Graduada no se podía hacer esa importante aportación, y aunque existía una legislación específica (desde 1898), el desarrollo de este movimiento no fue real y se limitó a su aplicación en determinados lugares, no de una forma generalizada.

Por ejemplo, se puede mencionar la situación de Madrid, donde a la insuficiencia de presupuestos municipales se unió que no existían los nuevos locales

6 Antonio Ballesteros, La escuela graduada, Madrid: Publicaciones de la Revista de Pedagogía, 1926. 
necesarios ${ }^{7}$. También añadir que a los maestros se les pagaba la vivienda y en un breve espacio de tiempo se crearon 128 plazas de maestros que no tenían residencia ni lugar donde trabajar. En definitiva, hubo una acusada descoordinación entre las administraciones estatal y municipal, que no estaban suficientemente concienciadas de la importancia real de favorecer un desarrollo de la educación.

Existían problemas legales, financieros o corporativo-grupales, pero también había ciertas "inercias y resistencias mentales, residuos del pasado, comprensiones de una realidad a partir de percepciones y esquemas ya obsoletos" ${ }^{8}$. Por tanto, la dificultad de esta tarea no residía únicamente en la falta de medios, sino la complicación de cambiar en los docentes la mentalidad y la necesidad de trabajar en equipo, coordinándose con los demás compañeros.

También se dieron situaciones no esperadas, como problemas para el control de tantos alumnos en un mismo centro, aspecto que llevó a que en algunos momentos se guardaba más parecido con una estructura militar que con un centro educativo.

En definitiva, se busca una escuela moderna y adaptada, con nuevos valores de renovación de la enseñanza. Puede parecer que simplemente se pretendían algunos cambios más concretos, pero abarcaba una reforma muy profunda que intentaba variar el concepto de enseñanza en global, ampliando la educación a todos los sectores de la sociedad. Se puede vislumbrar con claridad la vigencia de todos estos valores y planteamientos al revisar el actual modelo educativo.

\section{La Escuela Graduada en el actual marco educativo}

Muchos aspectos supusieron una innovación en esa época, que son o han sido fundamentales en sistemas educativos posteriores e incluso el actual.

Uno de los pilares básicos de la Escuela Graduada es la clasificación de alumnos en grupos homogéneos, que permitan un trabajo más específico, sin necesidad de atender a un amplio abanico de edades, y por tanto distintas características, necesidades, perspectivas, etc. Sin embargo fue muy criticada la manera de establecer esas clasificaciones. A finales del siglo xIx y principios del xx se da un desarrollo muy importante de técnicas de psicología experimental. Aparecen los primeros test standard que permiten agrupar a alumnos según su coeficiente intelectual, tarea en la que destacaron investigadores como Cattel o Binet.

Vistos de una forma general, los principales elementos que se añadieron al campo educativo o sufrieron una importante transformación, cambiando sustancialmente la primera enseñanza, fueron los siguientes:

- Programas

- Libros de texto y enciclopedias

- Extensión del currículo, nuevas materias

7 María del Mar Pozo, Urbanismo y educación. Política educativa y expansión escolar en Madrid (1900-1931). (Alcalá de Henares: Universidad de Alcalá, 1999).

8 Antonio Viñao, Innovación pedagógica y racionalidad científica. La escuela graduada pública en España (1898-1936) (Madrid: Akal, 1990). 
- Clasificación y distribución de niños

- Graduación del currículo

- Distribución de tiempos y tareas

- Evaluación y promoción o repetición

Los elementos que se destacan son la clasificación y evaluación del alumnado. Ya se ha comentado que los avances de las ciencias experimentales influyeron bastante en la educación de la época. Hasta ese momento, el concepto de evaluación se podía concebir como una valoración de las capacidades y trabajo del alumno a lo largo del curso. Sin embargo ahora no solo cambiaba el significado, sino su distribución en el tiempo. A la hora de clasificar a los alumnos según su diversidad se podía hacer por edad o por capacidad, donde se situaba la evaluación como elemento de categorización en el comienzo del proceso. Por tanto, actualmente el proceso evaluativo se sucede (por lo menos) al principio y al final de la enseñanza.

Otra particularidad interesante fue la introducción de enseñanzas de aplicación o complementarias. Además de las áreas de una mayor carga académica, se pretendió dar salida a otros aprendizajes más prácticos que fueran orientados a la unión de la enseñanza primaria y la profesional. Se podría considerar como la primera proyección de la formación profesional en todas sus variantes que se han dado en los últimos años. Así se favorecía una formación más completa a través de talleres de trabajos manuales o los campos de experiencias agrícolas y jardinería. Todo esto, evidentemente, estaba previsto en la construcción de los nuevos edificios, habilitando los espacios necesarios para cada caso. Además, se constituyó una oficina de orientación profesional que pretendía favorecer el paso del terreno académico al laboral. Este avance confirió otro valor a la educación, donde no solo se preparaba a los educandos en el terreno académico, sino que amplió el campo de la enseñanza con otros horizontes distintos, donde se atendía en definitiva a la diversidad de individuos de una sociedad cambiante y que exigía una educación cada vez más amplia y favorecedora de la igualdad.

Se pueden encontrar muchos criterios educativos en materia de metodología y organización en el aula. En su momento supusieron un pilar importante para las nuevas propuestas, basadas en sistemas del viejo continente que durante el siglo xix desarrollaron nuevas ideas basadas en autores como Rousseau o Pestalozzi. Entre los principales criterios vale citar los siguientes:

- El horario de mañana debe comenzar con trabajos más intensos (período de mayor rendimiento) y terminar con los más fáciles (comienzo de la fatiga).

- Después de un trabajo de abstracción se debe pasar a otro de tipo manipulativo.

- Para hacer desaparecer la fatiga, utilizar actividades más lúdicas y que no exijan una gran carga mental (adaptar las actividades dependiendo del momento del día). 
- Duración creciente de los descansos a lo largo de la jornada escolar, de forma que se permita un reposo adecuado que favorezca el rendimiento académico en otros periodos de tiempo.

- Se fomenta la especialización en un grado, de igual forma que en Educación Infantil y Primaria se favorece la permanencia del mismo maestro durante dos o tres cursos (Loe 2/2006, del 3 de mayo).

Así queda patente la vigencia de algunos aspectos de cierta relevancia, ya sea por la aparición de la Escuela Graduada o por una progresiva evolución de la metodología docente que se utiliza en el campo educativo.

No es un hecho aislado, sino que a lo largo de la historia de España se han producido muchos cambios políticos que, por supuesto, han repercutido en el planteamiento educativo. En multitud de ocasiones se ha usado la educación como instrumento político más que como un elemento de desarrollo de la sociedad. Así podemos mencionar muchos periodos políticos (dictadura de Franco, la Segunda República, dictadura de Primo de Rivera o el sexenio revolucionario, con gobiernos de Serrano, de Saboya o de la Primera República), que no han permitido dar la necesaria continuidad a los cambios y evolución desde el punto de vista educativo.

En España hubo una serie de circunstancias que implicaron la dificultad de implantar la Escuela Graduada, tal como se comentó, pero no sucedió de igual forma en otros países. Se puede mencionar el ejemplo prusiano, donde los centros educativos estaban dotados con un gran número de maestros, ayudantes. De esta forma se pudieron establecer distintos niveles o grados a los niños con los recursos adecuados 9 . Si se analizan los planteamientos organizativos de la escuelas graduadas madrileñas al principio del siglo $\mathrm{xx}$, por ejemplo, se pueden encontrar deficiencias y problemas en la distribución de tiempos, organización de contenidos y programas, uso de libros de materiales escritos o la aplicación de las metodologías innovadoras ${ }^{10}$. El principal problema que hubo en Madrid u otras zonas de España para el desarrollo de la Escuela Graduada (falta de profesorado), en otros lugares se solventó debido a los medios disponibles. Esto hace pensar que, con los recursos adecuados, se podría haber desarrollado más ampliamente este modelo.

Se puede concluir que la Escuela Graduada no responde simplemente a la graduación u ordenación educativa, sino que abarca un campo más amplio. Se busca una reforma educativa para adecuar la educación al nuevo contexto europeo. Sin embargo es posible comparar a la Ley Moyano de 1857, en el aspecto de que buscaba una reforma (tal vez demasiado ambiciosa para la época y recursos), que no se pudo llevar a cabo por las distintas críticas y oposiciones que ya se han comentado.

9 Antonio Viñao, "La Renovación de la Organización Escolar: La Escuela Graduada", en Psicología y pedagogía en la primera mitad del siglo XX, ed. por Javier Sáenz (Madrid: UNED, 2003), 73-104.

10 María del Mar Pozo, "La escuela graduada madrileña en el primer tercio del siglo XX: ¿un modelo pedagógico para el resto del estado español?”, Revista Complutense de Educación, 7, n. ${ }^{\circ} .2$ (1996): 237-241. 


\section{Organización escolar}

La clasificación y distribución de los niños planteó desde el principio un debate entre homogeneidad y heterogeneidad de las clases. Este planteamiento ponía en peligro que se olvidara la atención individual a las necesidades de cada niño. Al agrupar a los alumnos se debatieron los criterios a seguir, siendo los principales la edad biológica, el nivel de conocimientos (en especial la lectura y escritura) o la edad mental. El primero ofrecía menos dudas, mientras que los otros dos requerían la elaboración y aplicación de pruebas o tests específicos a los alumnos.

Hasta ahora se han comentado las características, problemas y criterios novedosos de la Escuela Graduada. Se aportarán a continuación algunos argumentos pedagógicos que ayudaron a promover la renovación del sistema educativo y repercutieron directamente en la revisión y desarrollo de una nueva organización escolar.

Es indudable la influencia que tuvo la Escuela Graduada en el sistema educativo español durante las primeras décadas del siglo xx, y en aquella época se planteó como la única opción de impulsar una educación integral, completa y moderna. Uno de los autores que defendió a ultranza esta opción fue Manuel Bartolomé Cossío a principios del siglo xx. Consideró que era necesaria y urgente la agrupación por edades y grados de cultura para formar verdaderas clases homogéneas. Además, la enseñanza de una amplia diversidad de alumnos en la misma aula (Escuela Unitaria) la calificó de "deplorable" y "antipedagógica mezcolanza de niños" ${ }^{\prime 1}$.

El desarrollo de distintas técnicas de la pedagogía científica, tan en liza en aquel momento, favoreció la evolución de la educación en general e impulsó modelos distintos a los vigentes. Se contribuyó entre otros logros a extender la enseñanza obligatoria, que se presentaba como necesaria al comparar el desarrollo educativo de España con los del resto de Europa. En un intento de clasificar y establecer las diferencias entre los alumnos se empezaron a introducir los exámenes escritos como un instrumento estandarizado. Incluso en la década de los sesenta se generalizaron pruebas oficiales de promoción.

Sin embargo, este afán de aglutinar muchos alumnos en los centros escolares creó numerosos problemas de organización, dando al director y a los profesores, en ocasiones, funciones autoritarias. Por tanto se trabajó duramente en marcar en cada escuela una misma línea de actuación que favoreciera la vigilancia de un gran número de alumnos. También hay que tener en cuenta que ese control no se limitaba a los alumnos, pues la nueva figura del director implicaba la supervisión de la forma de actuar de los profesores. Por tanto se creaban distintos rangos y conflictos internos entre los profesores y aunque tenían libertad de cátedra, perdían la independencia con que habían trabajado hasta entonces.

11 Antonio Viñao, "El maestro, lo que más importa. Cossío y las escuelas graduadas de Cartagena (1900)", Bordón. Revista de pedagogía, n. ${ }^{\circ} 258$ (1985): 413-420. 
Este modelo permitió asegurar la acción directa, sin intermediarios, del maestro sobre el niño, sin el recurso a los monitores, alumnos de más edad o los maestros auxiliares, metodologías propias de la enseñanza mutua o de la unitaria. De esta forma satisfacían las demandas y necesidades de escolarización de aquella época. Se tuvieron que abordar problemas como la aplicación de distintas políticas y respuestas organizativas, así como la relación entre educación y urbanismo ${ }^{12}$.

Antes de crear Escuelas Graduadas, propiamente dichas, hubo formas intermedias entre las que destacamos el "sistema inglés" (aulas separadas e independientes) y el sistema prusiano (aulas conectadas o separadas por cortinas $\mathrm{u}$ otro sistema). Más adelante, en la medida que los medios materiales permitían la construcción de un nuevo recinto escolar, en España se optó principalmente por el modelo panóptico (Imagen 1), que facultaba al director controlar el resto de aulas desde la posición central del colegio y así llevar a cabo las nuevas funciones que se le agregaban ${ }^{13}$ (Foucault, 1986).

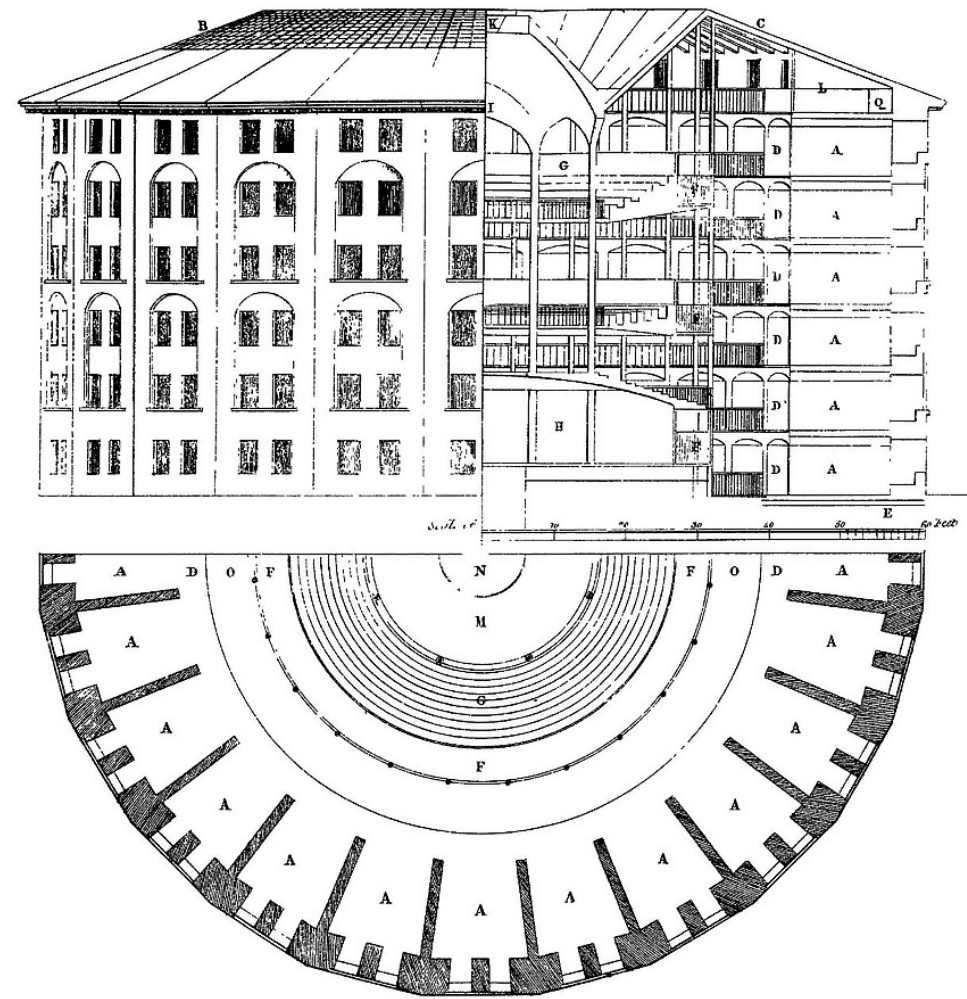

Modelo panóptico de Bentham. Fuente: Foucault (1986).

12 Claudio Lozano, Historia de la educación latinoamericana (Buenos Aires: Miño y Dávila, 2012).

13 Michel Foucault, Vigilar y castigar (Madrid: Siglo XXI, 1986). 
Después de todas las bases teóricas que se pusieron de manifiesto en España a partir de 1898, se encuentra que a la hora de ponerlas en práctica la graduación de la enseñanza a nivel organizativo no surtió efecto. Así tenemos que en la década de los años veinte y treinta se podían encontrar en la misma clase alumnos de cinco a seis edades distintas. También se unían los que llegaban al principio del curso, a medias y los más retrasados o repetidores.

Esta situación, y otras ya comentadas, dejaban un panorama difícil para la aplicación de las propuestas de este modelo y así se produjo en esas décadas una crisis ideológica, teórica y práctica, rompiendo la supuesta homogeneidad entre los alumnos, por lo que al final la actuación del maestro era similar o muy parecida a la realizada por un docente en una escuela unitaria.

\section{Espacios escolares en la Escuela Graduada}

Los recursos humanos son trascendentales en cualquier modelo educativo que se haya implantado a lo largo de la historia. Sin embargo, también se debe hacer mención al edificio en el que se lleva a cabo la actuación docente. Se puede encontrar alguna referencia bibliográfica de la época que refleja la importancia de las instalaciones en este modelo ${ }^{14}$. La disposición y ubicación física de los distintos espacios que se destinan a alguna finalidad o función concreta dentro de una institución, reflejará la importancia del papel y tareas asignadas a esta función ${ }^{15}$. En la estructura de la Escuela Unitaria no era tan relevante la organización de los recursos espaciales, pues la actividad se concentraba en un aula principal. Aunque la realización de distintas tareas, dadas las diferencias de los alumnos, podía implicar una estructuración diferente. El nuevo modelo graduado supuso una reorganización mucho más compleja, pues la distribución de varias aulas (dependiendo del número de grados), de la biblioteca, comedor o el campo escolar para experiencias agrícolas, exigía la inclusión de otros criterios, muy distintos al modelo anterior.

Es interesante analizar los espacios escolares para así ver la importancia que se les ha atribuido en cada periodo. La educación adquiere mayor relevancia en 1869 , cuando por primera vez se intenta llevar a cabo un programa de construcción de escuelas de enseñanza primaria. Hasta entonces, los centros educativos habían tenido carácter provisional, lo que deja claro la atención que se les otorgaba.

A partir de esta época se empezaron a construir edificios específicos con finalidades educativas. Estos diseños propios incluían una serie de dependencias, de las cuales dos tuvieron especial relevancia en cuanto a la polémica creada: la biblioteca y la capilla.

La primera se empezó a proponer a partir de 1869, sobre todo por el gobierno revolucionario que pretendía crear las llamadas "bibliotecas populares".

14 Luis A. Pelliza, "Escuela graduada: Memoria - 1920", Humanidades, n. ${ }^{\circ} 1$ (1921): 564-579.

15 Antonio Viñao, "Espacios escolares, funciones y tareas: La ubicación de la dirección escolar en la escuela graduada", Revista Española de Pedagogía, n. 228 (2004): 279-304. 
Los arquitectos le quisieron dar una posición central y con fácil acceso desde el exterior, para que tuviera la función comentada. La concepción de la biblioteca como recurso educativo abría muchas puertas como elemento que atendía a la diversidad del alumnado. Ya sea en el modelo unitario o en el graduado, hay que tener en cuenta que no todos los educandos trabajan al mismo ritmo. A pesar de los intentos de homogeneizar las clases con alumnos del mismo nivel, es necesario recurrir al trabajo individualizado pues los distintos ritmos y rendimientos de cada individuo necesitan recursos que favorezcan el desarrollo personal. Y es aquí donde juega un papel muy importante la biblioteca dentro del centro escolar. Pero debido a los problemas expuestos no fue posible generalizar esta dependencia dentro de las escuelas. Posteriormente, tras la aprobación de la Ley General de Educación de 1970, hubo otro intento de configurar bibliotecas escolares, pero tampoco llegó a buen puerto.

En cambio, la implantación de la capilla en los centros docentes sí se llegó a materializar a partir de los años cuarenta. Más adelante vería modificada su función, convirtiéndose en un salón de actos en la década de los ochenta, una vez se modificó el contexto ideológico-político en que había sido creada. Es paradójico que tuvo más relevancia la consolidación de un espacio que nada tenía que ver con el ámbito académico.

Otros espacios que tuvieron más o menos importancia en este marco fueron los aseos, la sala de profesores, la sala de alumnos, la sala de visitas, el gimnasio, el gabinete médico o los laboratorios; también los talleres de trabajos manuales y los campos de experiencias agrícolas y jardinería. La introducción de estos espacios en el centro educativo permitía la formación del alumno en una propuesta menos académica, pero igualmente válida y respetable, donde se ofrecía una mayor variedad de oportunidades, y que permitía adaptarse a una diversidad más amplia de alumnado, abriendo la educación a todos los sectores de la sociedad.

Respecto a la dirección escolar, no hay ningún documento escrito que haga referencia a la importancia de esta dependencia. Sin embargo, podemos analizarlo a partir de su existencia o no, su ubicación, su relación o vinculación con otros espacios, su accesibilidad o su dimensión. Sobre la base de estos criterios encontramos una concepción minimalista, que implicaría la inexistencia de un espacio específico para esta tarea o muy reducido. También hubo una noción maximalista, principalmente en colegios privados o de élite, donde el despacho suele hallarse en un lugar privilegiado.

Estos modelos serán adoptados en los centros dependiendo de la defensa de la Escuela Graduada o de preservar a ultranza el modelo unitario. Éste último tenderá a quitarle importancia a la función directiva, mientras que la primera le proporcionará una posición de favor, como un importante elemento dentro del nuevo modelo en auge. Esta situación se dio al menos los primeros años (desde 1898), pues la confusión y el vacío legal creaba dudas acerca de la función directiva. 
En cuanto a las propuestas de distribución de espacios en España, las primeras que se encuentran son de Rufino Blanco y Francisco Ballesteros. El segundo concede más importancia a la dirección escolar, pues Blanco se reserva en sus propuestas una visión minimalista, sin destinar ningún espacio específico. Éste último se valía del aula central para realizar las funciones como director del centro. La ubicación de esa dependencia le permitía realizar su actividad docente con los alumnos de último curso y a la vez controlar el resto de unidades a través de las cristaleras que se colocaban en los modelos panópticos de la Escuela Graduada.

Estas nuevas arquitecturas fueron destinadas a favorecer el desarrollo educativo dentro del centro, y también para permitir la apertura de los colegios a otro sector que cada vez será más relevante: las familias. Por tanto, se le va dar más importancia al entorno, considerando en la educación de los alumnos otra variable significativa que hasta entonces no se había tenido en cuenta, el ambiente donde se desarrolla. Todo esto repercutirá en una mejor atención a las características del alumnado.

\section{Escuela Unitaria versus Escuela Graduada, una experiencia}

Durante las primeras décadas del siglo xx estaba en pleno desarrollo la Escuela Graduada, en contraposición a la tradicional Escuela Unitaria. Hubo algún autor, como Santiago Hernández Ruiz, que mostró siempre su inclinación por la segunda en el avance educativo en países en vías de desarrollo de Sudamérica. Este pedagogo, en sus propias palabras, explica

cómo el pensamiento y experiencia de un educador formado en las corrientes pedagógicas innovadoras que circulaban en la España de los años veinte y treinta se integrarían en los nuevos discursos del desarrollismo y la planificación educativa, iniciados en la segunda posguerra mundial y dirigidos a los países entonces bautizados de subdesarrollados ${ }^{16}$.

El panorama que se encontró era un continente en su mayoría subdesarrollado, que intentaba mejorar considerablemente la educación y extenderla a todas las zonas que fuera posible. Sin embargo, esta expansión educativa estaba orientada desde lo cuantitativo, por lo menos en un primer momento. Hernández criticó duramente este aspecto pues no era partidario de esta orientación economicista, de buscar una rentabilidad financiera a partir de la educación. Fue nombrado experto itinerante de la Unesco, organismo que impulsó una política de equiparación de oportunidades educativas en la educación primaria, tanto en la duración de la escolaridad, como al nivel de los estudios realizados.

Una de las medidas que se introdujeron en la década de los cincuenta fue el libro de texto gratuito y único, como un primer intento de implantar la igualdad en la

16 Gabriela Ossenbach Sauter, “La labor de Santiago Hernández Ruiz como experto de la Unesco en América Latina, 1959-1966”, en Santiago Hernández Ruiz (1901-1988) y la educación de su tiempo, eds. Alejandro Tiana y Víctor Manuel Juan (Zaragoza: NED-Calatayud, 2002), 253-276. 
educación, ambicionando extender la enseñanza primaria al mayor sector de población que fuera posible.

Respecto a la población, había una gran parte que residía en zonas rurales, donde tenía cabida únicamente la Escuela Unitaria ${ }^{17}$. Sin embargo, se pretendía que ese fuera el camino a seguir. Se criticaba abiertamente la graduación de la enseñanza, las técnicas de evaluación y la promoción de los alumnos, estableciendo como un mito la "homogeneidad de los grupos". Incluso comenta que no debe haber diferencias en el mundo rural y el mundo urbano.

Fue este aspecto de la homogeneidad el que más se criticó a la Escuela Graduada, en ascenso por aquella época. Se manifestaron opiniones bastante severas, como que "ante el dogma de la homogeneidad, la escuela unitaria sólo puede ser condenada al infierno. Porque es la imagen pura de la heterogeneidad". También afirma, refiriéndose a la mentalidad de escuela graduada, que "los actuales homogeneizantes radicales representan la reducción de esa mentalidad a términos de hipérbole, pero no la han inaugurado" ${ }^{\prime 18}$.

Aparece un concepto muy criticado, "el fracaso escolar". La transformación de los sistemas de promoción y evaluación de los alumnos provoca la aparición de esta noción. Representa un conjunto de individuos que, en principio, no mantienen la homogeneidad del grupo de similares características, propician la aparición de la diversidad. Todo esto desemboca en la repetición de cursos, desmotivación por parte del discente y abandono de la escuela.

En contraposición a las clasificaciones rígidas de la escuela graduada, se proponen otras líneas de actuación más flexibles. Se busca que no pierdan la heterogeneidad que constituye la naturaleza de la escuela unitaria, criticando las evaluaciones y ordenamientos realizados por la administración. En definitiva, es un discurso muy distinto al de la evaluación y calidad de la enseñanza que circulaba por aquellos años en boca de otros expertos educativos. Se califican estas actuaciones como "paidófobas" y "puerívoras".

La Escuela Unitaria mantiene aún sus fieles seguidores, defendiendo que sólo es posible desarrollar a través de ésta, la iniciativa personal de los alumnos, el trabajo colectivo e individual. Este modelo ofrece interesantes posibilidades de desarrollo, favorece el trabajo autónomo o rompe barreras generacionales. Este aspecto se criticó posteriormente, pues si la heterogeneidad aporta beneficios a los alumnos, en las clases graduadas también la encontramos. Aunque los alumnos tienen la misma edad cronológica, la edad madurativa no es igual, ni psicológica ni fisiológica.

Hernández Ruiz considera que las palabras "adelanto" y "retraso" no se debían calificar globalmente en la escuela primaria, pues nadie es adelantado ni retrasado en todos los ámbitos. Un mismo alumno muestra diversidad en sus propias capacidades para cada materia. Dada la dificultad de medir estos distintos avances en cada materia y en cada alumno, se hace un alegato a favor de la pedagogía, reprochando a la psicología el establecimiento de unos criterios de evaluación del rendimiento escolar, por lo menos en la enseñanza primaria.

17 Alba Triana, "Formación de maestros rurales colombianos 1946-1994", Revista historia de la educación latinoamericana, 14, n. ${ }^{\circ} 18$ (2013).

18 Santiago Hernández, La escuela unitaria completa (La Habana: Centro Regional de la Unesco, 1961). 
Sin embargo, en la enseñanza secundaria, sí tenía planteamientos más cercanos a la Escuela Graduada, debiendo regirse por un sistema de exámenes. Esta concepción es muy distinta a la que se promueve en la enseñanza primaria, donde debe primar la idea del niño como hombre en formación, ajena a las ideas de éxito y fracaso, donde se favorezca la idea de igualdad de oportunidades para todos los individuos, ya destacada en la Constitución Española (CE, 1978) y en los principios de la legislación actual (art. 1 LOE).

Estas teorías pueden tener su significación en aquella época y aquel lugar. Pero después de muchos años hay que rendirse a la evidencia: la graduación es el sistema que se ha implantado en prácticamente todo el mundo. Sin embargo, tenemos en cuenta que en el presente a veces se recurre al sistema de la Escuela Unica, como en zonas con baja población (Centros Rurales Agrupados), donde incluso se llegan a reunir niños de varias aldeas para agrupar un aula unitaria. Este caso se presenta en muchos lugares remotos de España. También tenemos la alfabetización de adultos, que incluye a alumnos de varias edades en una misma aula.

En el caso concreto de Hernández Ruiz, apoya la fusión de ambos modelos con un carácter aperturista. No obstante, dada la situación que encontró al llegar a Latinoamérica, no es de extrañar que se volcara en defensa de la Escuela Unitaria, pues el panorama era el de un gran número de escuelas que solo impartían dos o tres niveles y se quedaban a medias de este modelo y el graduado. Hay que tener también en cuenta la falta de recursos humanos. Esta misma situación se dio en España a principios del siglo xx y desembocó en la imposibilidad de implantar la Escuela Graduada.

La defensa e implantación del modelo unitario de enseñanza no era el objetivo, sino que esta lucha tuviera otra razón de fondo, como es la reivindicación de una mayor importancia del maestro y la pedagogía. Se demanda en muchas ocasiones la mejora de la educación, desde una visión de arte y vocación, rechazando la cuantificación y excesiva estructuración de una labor tan abstracta y complicada, que muchas veces huye de toda regla.

Respecto a la instrucción del profesorado, los maestros de modelos unitarios y de graduados debían instruirse del mismo modo, pues "en su formación debía adquirir una actitud abierta, no adherida férreamente a una metodología, que le permitiera adaptarse a las condiciones particulares de cada situación" ${ }^{\prime 19}$.

Por último, comentar que estos planteamientos, a pesar de los años transcurridos, están a la orden del día, y seguramente permanecerán durante mucho tiempo. Desde la aplicación de las técnicas experimentales en la educación se ha tendido a valorar el aspecto cuantitativo de la educación, aumentando los procesos de evaluación y mejora de la calidad de la enseñanza. Sin ir más lejos, podemos fijarnos en planteamientos que surgieron aproximadamente a mediados del pasado siglo (con las reválidas) y las evaluaciones que se pretendían imponer con la LOMCE. Pero esta tendencia hay que tomarla con cautela pues debemos tener presente la dificultad de evaluar la educación (sobre todo en la enseñanza primaria), dado que ésta comprende mucho más que la mera medición de la competencia de los alumnos en una serie de destrezas o aprendizaje de contenidos.

19 Ossenbach, op. cit. 
Es remarcable que en la actualidad la Escuela Graduada es el modelo más implantado, pero en algunas zonas todavía se mantiene la dualidad ya comentada. En el caso de México, las escuelas unitarias representan más del cuarenta por ciento en las escuelas públicas. Esta persistencia a lo largo de todos estos años se puede deber a las condiciones del mundo rural y su trayectoria histórica, donde a aportación de soluciones pedagógicas es limitada ${ }^{20}$.

\section{CONCLUSIÓN}

En el presente artículo se ha pretendido ofrecer una visión general de la innovación que supuso la aparición de la Escuela Graduada en el panorama educativo de finales del siglo xIx y principios del siglo xx. Durante esas décadas, surgieron muchas reticencias ante la inclusión de nuevas concepciones pedagógicas que suponían un cambio tan sustancial con el modelo educativo imperante, la Escuela Unitaria. Además, el contexto económico y social no ayudó a implantar modificaciones tan notables. Sin embargo, se puede apreciar en la lectura del artículo que muchos de los planteamientos actuales tienen un reflejo claro en los años en que se implantó la Escuela Graduada. Se hacen referencias a la legislación actual y el existente modelo pedagógico para presentar las similitudes entre dos épocas muy distintas, tanto en el plano político como social. Pero se encuentran muchos puntos de unión, y queda de manifiesto que las corrientes actuales mantienen analogías directas a pesar de ser un contexto muy diferente. Se ha pretendido realizar un repaso de las principales características de la Escuela Graduada en cuanto a su estructura, recursos y organización. Se finaliza con una comparación de este modelo y el Unitario según una experiencia en la América Latina, que era el más extendido en aquella época, y como se menciona en el artículo, sigue vigente en muchas zonas de Sudamérica.

\section{FUENTES}

Constitución Española. Boletín Oficial del Estado, 29 de diciembre de 1978, n. ${ }^{\circ}$ 311, pp. 29313 a 29424.

Ley Orgánica 1/1990, de 3 de octubre, de ordenación general del sistema educativo. Boletín Oficial del Estado 4 de octubre de 1990 [consultado el 16 de octubre de 2017]. Disponible en: http:// www.educacion.gob.es/mecd/oposiciones/files/logse.pdf

Ley Orgánica 2/2006, de 3 de mayo, de Educación. Boletín Oficial del Estado, 4 de mayo de 2006, n. ${ }^{\circ} 106$ [consultado el 16 de octubre de 2017]. Disponible en: https://www.boe.es/buscar/ pdf/2006/BOE-A-2006-7899-consolidado.pdf

Ley Orgánica 8/2013, de 9 de diciembre, para la mejora de la calidad educativa. Boletín Oficial del estado 10 de diciembre de 2016, número 298 [consultado el 16 de octubre de 2017]. Disponible en: https://www.boe.es/buscar/pdf/2013/BOE-A-2013-12886-consolidado.pdf

Ministerio de Instrucción Pública y Bellas Artes. Planos modelos de escuelas graduadas con presupuestos reducidos. Madrid: Imprenta del Colegio Nacional de Sordomudos y Ciegos, 1912.

20 Elsie Rockwell y Garay Claudia, "Las escuelas unitarias en México en perspectiva histórica: un reto aún vigente", Revista Mexicana de Historia de la Educación II, n. ${ }^{\circ} 3$ (2014): 1-24. 


\section{REFERENCIAS}

Ballesteros, Antonio. La escuela graduada. Madrid: Publicaciones de la Revista de Pedagogía, 1926. Foucault, Michel. Vigilar y castigar. Madrid: Siglo XXI, 1986.

Guil, Ana y Sara Vera. "Entre Europa y América Latina: Mercedes Rodrigo, psicopedagoga pionera". Revista Historia de la Educación Latinoamericana, 13, n. ${ }^{\circ} 17$ (2012): https://doi. org/10.19053/01227238.1598.

Hernández, Santiago. La escuela unitaria completa. La Habana: Centro Regional de la Unesco, 1961.

Lozano, Claudio. Historia de la Educación Latinoamericana. Buenos Aires: Miño y Dávila, 2012.

Moreno, Pedro L. "Renovación pedagógica y compromiso social en la edad de plata de la Pedagogía española: Félix Martí Alpera (1898-1920)". Revista española de pedagogía, 63, n. 231 (2005): 203-222.

Orden, Arturo de la. “La escuela en transición”. Revista de educación, n. ${ }^{\circ} 266$ (1981): 133-149.

Ossenbach Sauter, Gabriela, "La labor de Santiago Hernández Ruiz como experto de la Unesco en América Latina, 1959-1966". En Santiago Hernández Ruiz (1901-1988) y la educación de su tiempo, eds. Alejandro Tiana y Víctor Manuel Juan, 253-276. Zaragoza: UNED-Calatayud, 2002.

Pelliza, Luis A. “Escuela graduada: Memoria - 1920”. Humanidades, n. 1 (1921): 564-579.

Pozo, María del Mar. “La Escuela Graduada Madrileña en el primer tercio del siglo xx: ¿Un modelo pedagógico para el resto del Estado español?". Revista Complutense de Educación, 7, n. ${ }^{\circ} 2$ (1996): 237-241.

Pozo, María del Mar. Urbanismo y educación. Política educativa y expansión escolar en Madrid (19001931). Alcalá de Henares: Universidad de Alcalá, 1999.

Rockwell, Elsie y Claudia Garay. “Las escuelas unitarias en México en perspectiva histórica: un reto aún vigente". Revista Mexicana de Historia de la Educación, II, n. ${ }^{\circ} 3$ (2014): 1-24.

Triana, Alba. "Formación de maestros rurales colombianos 1946-1994". Revista Historia de la Educación Latinoamericana, 14, n. $^{\circ} 18$ (2013).

Viñao, Antonio, "La renovación de la organización escolar: la Escuela Graduada". En Psicología y pedagogía en la primera mitad del siglo XX, ed. Javier Sáenz, 73-104. Madrid: UNED, 2003.

Viñao, Antonio. "El maestro, lo que más importa. Cossío y las escuelas graduadas de Cartagena (1900)". Bordón. Revista de pedagogía, n. 258 (1985): 413-420.

Viñao, Antonio. "Espacios escolares, funciones y tareas: La ubicación de la dirección escolar en la escuela graduada". Revista Española de Pedagogía, n. 228 (2004): 279-304.

Viñao, Antonio. Innovación pedagógica y racionalidad científica. La escuela graduada pública en España (1898-1936). Madrid: Akal, 1990.

Cómo citar:

Navarro Martínez, Óscar. "La Escuela Graduada en España. Similitudes con el actual modelo educativo" Revista Historia de la Educación Latinoamericana. Vol. 20 No. 31 (2018): 211-228

DOI: $10.19053 / 01227238.7394$ 